\title{
20 Jahre BIOspektrum - ein BIOtorial zum Jubiläum
}

DOI: $10.1007 / \mathrm{s} 12268-015-0524-8$

(C) Springer-Verlag 2015

Bis auf das mit Urknall explodierte All hat alles auf der dabei entstandenen Welt eine mehr oder weniger lange Vorgeschichte. Wir vermessen sie als zehnfingerige Wesen in Dekaden, deren Teil oder Vielfaches - wohlwissend, dass das Zwölfersystem aus der Stadt Ur mathematisch praktischer wäre. Aber die Evolution ist eine Bastlerin, keine Informatikerin. Zwar sind dies ihre NutznieBer (natürlich: User), die jedoch in Femtozeiten rechnen, für die uns wiederum die Natur, sowenig wie für die Entropie, einen Sinn entwickelt hat. Wir wollen nicht darauf warten, sondern Feste in Humandimensionen feiern. So also nun auch das Zwanzigste unseres BIOspektrum, das zwar nicht Weltgeschichte machen, aber doch ordentlich die sich expandierende bunte BIOgemeinde zusammenhalten will.

In diesem Alter ungefähr konnte man vor dem Zeitalter des lebenslangen Lernens eine für das Leben geltende Bildung vorweisen und damit ein Recht auf volle Partizipation am und im Staat beanspruchen. Wenn auch dies kaum mehr stimmt, wie alles andere (hinkt doch immer der Paragraf der Erfahrung nach), so freuen wir uns denn des „Zwanzigsten“ - nicht ohne einen dankbaren Rück- und scheuen Vorausblick.

Die alte Gesellschaft für Physiologische Chemie, gesamtdeutsch und altväterlich medizinisch-chemisch sozialisiert über die Runden der Gezeiten gekommen, hatte ihre Hauszeitschrift, den berühmten „HoppeSeyler“. Der namengebende Begründer und Netzwerker der quantifizierenden Physiologischen Chemie hatte das Fach durch Autorität und Eros geprägt und lange Jahre mit seiner Schulung gedeichselt. Seine Zeitschrift war zugleich Meilenstein und Siegel der Zugehörigkeit zum Clan, auch nachdem die „wahren“ (Naturstoff-, Enzym- und Reaktions-) Chemiker mit dem „Charisma“ der Nobel-
Koryphäen der 20. Jahrhundertwende konkurrierend vor die Mauern zogen. Die „biologisch-chemischen“ oder einfach „biochemischen“ Zeitschriften waren ihre Trompeten in England, Deutschland und den emanzipierenden USA. Verlagsinteressen hielten sie blank.

Zornige junge Leute freuten sich der stilistischen Freiheiten, der redaktionellen Entscheidungsgeschwindigkeit und der verlegerischen Gratisgaben, ja sogar Remuneration! Natürlich fehlten bunter Glanz und Anwendungsmoral von der Geschicht', aber auch stilistische Beckmesserei und nicht-informierende Langeweile. Und sie hatten einen offen zugänglichen ausgeführten Experimentalteil ohne Laborslang-Abkürzungen und GeheimSchibboleths.

Zugleich erweiterte sich die Biochemie und taufte sich um in die tönende Molekularbiologie und umfassendere Verzweigungen. Die Gemeinde und die Vielfalt wuchsen. Mit ihnen die Sozialknoten und Etikettierungen. Auch nachdem die DDR ihres Sonderweges gegangen war (ich vermisste die unprätentiösen Kollegen), blieb immerhin eine gemeinsame Großfamilie als GBCh. Ihre Routinegeschäfte wurden im „Hoppe-Seyler“ veröffentlicht. Ich dachte, eine Familienpostille mit persönlichen und wissenschaftlichen Mitteilungen aus Forschung und Beruf, Berufung und Preisung könnte den Zusammenhalt der Biochemiker aller Sparten festigen, etwa wie es die fest gestrickten Chemiker mit ihren Nachrichten aus der Chemie, den „Blauen Blättern“, halten, und erhielt grünes Licht. Deshalb und überhaupt kamen wir zu den „Grünen Blättern“ als eigener Beilage zum „Hoppe-Seyler“. Dass das etwas kosten könnte, hatte ich nicht bedacht, aber Dieter Oesterhelt, der damalige Chef, und Klaus Beaucamp, der Schatzmeister, machten helfende Miene zum Grünen Spiel, ich das Spiel, und es fand Zustimmung, sogar Interesse, als es 1994 aus Urgründen darum ging, vom Blättchen zum Blatt, zur veritablen Vereinszeitschrift zu kommen.
Das Plazet gewann der energisch-initiative Michael Weller vom Spektrum-Verlag. Titel und Aufmachung wurden auch entworfen, die VAAM mit im gemeinsamen Boot, und schließlich wurde eine junge, zielstrebige Dreidamen-Redaktion unter Frau Dr. Schreiber gesammelt. Seit 20 Jahren wurden also auch hier die „Gender“-Verhältnisse in aller Ruhe und Einvernehmlichkeit gekippt. Das zeigt sich u. a. auch bei den Redaktionskonferenzen, in denen vom hohen „Impact“ des BIOspektrum berichtet werden kann, das in seiner stetigen und soliden Art offensichtlich den Mitgliedern der fünf Gesellschaften verschiedener Nuancen der gleichen Couleur in bebildeter Aufmachung und aktueller Variation der Thematiken das Erwartete gibt.

Ein Dank hier auch vor allem den Bei- und Zuträgern aus ihrer aktuellen Forschung GBM, VAAM, GfG, DGPT und DECHEMA, in der Reihenfolge ihres Erscheinens. Brauchbare Späne der Tagesarbeit fallen nicht von alleine an. Sie müssen gesammelt und aufbereitet werden - Zeit, die der markensetzenden Konkurrenzforschung abgeht. Ein doppelt achtenswertes Opfer dem Gott des wissenschaftlich-sozialen Netzwerks, nicht bloßes inkontinentes oder selbstbefriedigendes Geschwätz für die „Wolke“.

Da kann man nur wünschen: „Weiter so“; als veramtlichter Nordrhein-Westfale natürlich mit „Glück Auf!“ - auf hundertzwanzig!

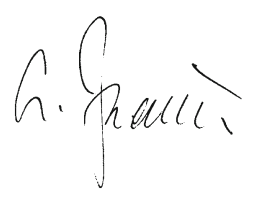

Lothar Jaenicke, Gründungsmitglied BIOspektrum, Universität zu Köln

Korrespondenzadresse:

Prof. Dr. Lothar Jaenicke Institut für Biochemie Universität zu Köln Zülpicher Straße 47 D-50674 Köln 